\title{
Atención de Pacientes en Tratamiento de Ortodoncia Durante la Pandemia COVID-19 (SARS-CoV-2). Presentación de un Algoritmo
}

\author{
Care of Patients in Orthodontic Treatment During the \\ Covid-19 Pandemic (SARS-CoV-2). Algorithm Presentation
}

\author{
Carolina Paz Matus Abásolo1; María Eugenia Nemeth Kohanszky² \& Montserrat Andrea Inostroza Tapia ${ }^{3,4}$
}

MATUS, A. C. P.; NEMETH, K. M. E. \& INOSTROZA, T. M. A. Atención de pacientes en tratamiento de ortodoncia durante la pandemia COVID-19 (SARS-CoV-2). Presentación de un algoritmo. Int. J. Odontostomat., 14(4):489-494, 2020.

RESUMEN: Los coronavirus son una familia de virus que se encuentran ampliamente distribuidos en la naturaleza en animales y humanos. El nuevo coronavirus (SARS-CoV-2) causa la enfermedad COVID-19, la cual se originó en Wuhan, China y se caracteriza por su alta tasa de contagio entre personas, por lo cual se declaró emergencia de salud pública de importancia internacional en enero de 2020 y pandemia global en marzo 2020, dada la alta propagación del virus a nivel internacional. Los pacientes que se encuentran en tratamiento de ortodoncia pueden presentar diferentes situaciones que requieran de atención por parte de la especialidad, suscitando un contacto cercano entre tratante y paciente, por ende, un alto e inminente riesgo de contagio por SARS-CoV-2. Ante esto, es fundamental para la práctica ortodóncica la implementación de protocolos de prevención, que permitan realizar inicialmente una correcta anamnesis del paciente vía remota, para establecer una clasificación de la urgencia, que en el caso de no poder ser diferida deberá realizarse bajo estrictas medidas de protección personal según el riesgo del paciente. El objetivo de este artículo es dar a conocer un algoritmo que entregue las recomendaciones actualizadas en la atención a pacientes en tratamiento de ortodoncia durante la pandemia COVID-19, a fin de disminuir la posibilidad de contagio y propagación de esta enfermedad.

PALABRAS CLAVE: COVID-19, Tratamiento ortodóncico, Ortodoncia, SARS-CoV-2.

\section{INTRODUCCIÓN}

Los coronavirus son una extensa familia de virus que pueden causar infecciones tanto en humanos como en animales. Las infecciones causadas por estos virus en el ser humano pueden ir desde un resfriado común hasta enfermedades más graves como el síndrome respiratorio de Oriente Medio (MERS-CoV) y el síndrome respiratorio agudo severo (SARS-CoV) (World Health Organization, 2020a).

EI COVID-19 es la enfermedad infecciosa causada por el coronavirus que se ha descubierto más recientemente (SARS-CoV-2), la cual era desconocida antes de que estallara el brote en diciembre de 2019 en Wuhan, China (Meng et al., 2020), detectándose en Chile el primer caso confirmado el 3 de marzo de
2020 (Ministerio de Salud, 2020a). Dentro de sus principales síntomas se encuentran fiebre, tos seca, disnea y cansancio general. Se estima que cerca de un $80 \%$ de las personas que padecen el virus se recuperan sin necesidad de tratamiento hospitalario. Sin embargo, aquellos con afecciones médicas previas como hipertensión arterial, problemas cardíacos o pulmonares, diabetes o cáncer están más propensos a desarrollar cuadros graves que pueden comprometer la vida (Gupta et al., 2020). Según los datos entregados por el Colegio Médico de Chile, la tasa de letalidad por coronavirus varía entre aproximadamente entre un $<1 \%$ y $7 \%$ dependiendo del país, de las medidas de salud pública implementadas, perfil demográfico y capacidad de respuesta del sistema de

\footnotetext{
${ }^{1}$ Cirujana Dentista, Facultad de Odontología, Universidad de Chile, Chile.

${ }^{2}$ Cirujana Dentista, Facultad de Odontología, Universidad del Desarrollo, Concepción, Chile.

${ }^{3}$ Especialista en Ortodoncia y Ortopedia Maxilofacial, Facultad de Odontología, Pontificia Universidad Católica de Chile, Chile.

${ }^{4}$ Especialista en Ortodoncia y Ortopedia Maxilofacial, Hospital Ricardo Valenzuela Sáez, Rengo, Sexta Región, Chile.
} 
salud (Colegio Médico de Chile \& Sociedad Chilena de Infectología, 2020), registrando Chile una de las cifras más bajas a nivel mundial con un 1,1\% (Ministerio de Salud de Chile, 2020).

Este nuevo virus se caracteriza por su alta tasa de contagio (superando al MERS-CoV y SARS-CoV). Su transmisión puede ser directa, principalmente a través de pequeñas gotas de secreciones respiratorias expelidas por algún individuo infectado y aerosoles generados durante procedimientos terapéuticos realizados a estos individuos o indirecta, mediante el contacto de las manos con superficies inanimadas contaminadas con estas secreciones, las que posteriormente pueden ser llevadas a las vías de entrada como mucosa oral, nasal y ocular (Guo et al., 2020; Volgenant et al., 2020). Lo anterior implica que actualmente el COVID-19 sea una pandemia que ha generado a nivel mundial, hasta el 19 de mayo de 2020 , un total de 4.731.458 casos confirmados y 316.169 personas fallecidas (World Health Organization, 2020b).

A la fecha, no hay evidencia científica que permita afirmar que algún tratamiento es efectivo y seguro contra el SARS-CoV-2, sin embargo, se encuentran en estudio diferentes medicamentos cuya utilidad aún es incierta (Colegio Médico de Chile \& Sociedad Chilena de Infectología), por lo que el propósito de controlar su diseminación se debe enfocar en las medidas preventivas. Estas incluyen: aislamiento social, control de la fuente infecciosa, detección temprana de pacientes contagiados y cese de la transmisión implementando protocolos de bioseguridad que consideran el uso de equipos de protección personal (EPP) para proteger al personal sanitario y a la población en general (Organización Panamericana de la Salud, 2020).

En la práctica odontológica habitual, los profesionales y pacientes se encuentran constantemente expuestos a agentes patógenos que habitan la cavidad oral y tracto respiratorio debido a la particular condición de generación de aerosoles, estrecha distancia de trabajo y uso de múltiples instrumentos e insumos. A su vez el cercano contacto con fluidos como la sangre y saliva que contienen una carga viral considerable en pacientes infectados, incluso en pacientes asintomáticos, conlleva un alto riesgo de infección por COVID-19 (Sociedad de Ortodoncia y Ortopedia Dentomaxilofacial de Chile, 2020).

Con el fin de optimizar las atenciones clínicas y no exponer innecesariamente a pacientes y funcionarios, se hace necesario gestionar las citaciones de los pacientes antes que acudan a la atención odontológica programada, aplazando todo procedimiento dental rutinario para priorizar la atención de las patologías incluidas en el GES de urgencias ambulatorias (Ministerio de Salud, 2020b).

Las recomendaciones para la atención de urgencia de especialidades odontológicas en fase IV COVID-19 (Ministerio de Salud, 2020b), considera asegurar acceso a las atenciones odontológicas catalogadas como críticas, es decir, cuadros clínicos que pueden agudizarse en el corto plazo (2 semanas) y los controles derivados de los cuadros clínicos antes mencionados. Dentro del área de ortodoncia, se sugiere previo a la atención del paciente, realizar un cuestionario que permita determinar si su diagnóstico coincide con el de una urgencia ortodóncica, definiéndose ésta como: un problema de salud oral que surge a raíz de un tratamiento ortodóncico, el cual necesita de una atención dental rápida para ser resuelto (Caprioglio et al., 2020). De esta manera, en el "Protocolo de bioseguridad para la atención en ortodoncia" sugerido por la Sociedad de Ortodoncia de Chile, se recomienda atender sólo urgencias en periodos de cuarentena a nivel nacional o local. Se considera lo siguiente como urgencias ortodóncicas calificadas:

1.Situaciones en las cuales el paciente presenta el desalojo total o parcial de algún elemento del tratamiento ortodóncico, el cual corre riesgo de ingerir o aspirar.

2.Lesiones en la mucosa oral debido a desajustes de aparatos ortodóncicos.

3.Traumatismos dentoalveolares en pacientes con aparatos ortodóncicos.

4.Abscesos o procesos infecciosos asociados a la aparatología ortodóncica (banda por ejemplo).

5.Paciente que presente alguna complicación por procedimiento quirúrgico reciente, relacionado a su tratamiento de ortodoncia.

En caso de que un paciente presente una urgencia ortodóncica calificada es necesario evaluar previamente si es posible que este pudiese recibir apoyo vía telefónica, videollamada o correo electrónico hasta que pase el periodo de pandemia. Si la urgencia es imposible de diferir, es necesario atender bajo un protocolo de bioseguridad, el cual debe ser conocido e implementado de manera estricta por el profesional.

El objetivo de este artículo es entregar las recomendaciones actualizadas para la atención de pacientes en tratamiento ortodóncico durante la pandemia 
COVID-19, a fin de disminuir las posibilidades de contagio en la práctica clínica y así contribuir y mejorar los niveles de control en la transmisión de esta enfermedad.

\section{RECOMENDACIONES PARA LA ATENCIÓN}

Manejo preliminar y clasificación del paciente. Debido a la suspensión temporal de los controles de rutina de los pacientes en tratamiento de ortodoncia, se recomienda a los especialistas mantener un contacto remoto con sus pacientes mediante las diferentes alternativas de comunicación que hoy en día existen, como diversas plataformas de consultas virtuales a distancia, teléfono de emergencia, correo electrónico o whatsapp, para así reforzar medidas preventivas y mantenerse al tanto de cualquier complicación que podría presentarse en el tratamiento, haciendo énfasis en lo siguiente:

1.El autocuidado de los aparatos ortodóncicos.

2.La correcta higiene oral y de los aparatos ortodóncicos (4 veces al día).

3. La mantención de una dieta saludable, evitando alimentos duros o retentivos.

4. El uso diario y manejo correcto de los aparatos removibles.

Si un paciente presenta alguna situación que altere el bienestar de su tratamiento ortodóncico, es aconsejable siempre el contacto remoto previo a la atención presencial, a modo de mantener seguros tanto al paciente como al personal tratante. Se recomienda realizar un TRIAGE vía telefónica entre el paciente (o sus padres) y un miembro del equipo de ortodoncia, quien identificará el problema y determinará si es o no una urgencia ortodóncica calificada y a su vez si amerita una consulta ortodóncica de urgencia.

Posterior al TRIAGE vía remota, si se confirma una urgencia ortodóncica calificada, se debe determinar la factibilidad de brindar apoyo e indicaciones para el manejo seguro por el paciente en su casa sin asistir a la consulta dental. De ser el caso, serán necesarias indicaciones claras y precisas a modo de evitar maniobras y procedimientos que puedan empeorar la situación. Por otra parte, si no es factible el manejo de la urgencia por el paciente de manera segura, ésta podrá ser atendida por el especialista, siempre y cuando durante la atención se cumplan los protocolos de bioseguridad de manera estricta.
Si bien, la Sociedad de Ortodoncia de Chile sugiere que "todo paciente debe considerarse como infectado", es fundamental realizar una correcta clasificación del riesgo potencial que posee de estarlo. Para ello se aconseja hacer uso de un cuestionario, el cual se envía al correo electrónico del paciente al momento de solicitar o confirmar la hora. En el cuestionario el paciente deberá contestar si o no, a una serie de preguntas sobre sus signos y síntomas de los últimos 14 días. Dentro de estos se considera la presencia de fiebre, cefalea, tos, disfagia, alteraciones del olfato y gusto, problemas respiratorios, mialgia y dolor abdominal. Además, deberá indicar si presenció eventos junto a 10 o más personas, si viajó fuera del país y si tuvo contacto con personas contagiadas por coronavirus. Si el paciente contesta de forma positiva a una o más preguntas es catalogado de alto riesgo.

Si el paciente posee una urgencia ortodóncica calificada, se debe evaluar la opción de diferir la atención y de otorgarle apoyo virtual, y en el caso de no ser posible se recomienda atender siempre bajo un protocolo estricto que se detallará más adelante.

\section{Manejo de urgencia ortodóncica calificada y aten- ción según riesgo de contagio}

Bajo riesgo. Una vez que el paciente asiste a la consulta se repite el cuestionario para corroborar el riesgo debiendo firmar un consentimiento informado, el cual certifica que está en conocimiento de la pandemia y voluntariamente consiente el tratamiento, considerando los riesgos.

Al momento de llegar a la consulta, el paciente debe realizarse un lavado de manos estricto con jabón o alcohol gel e idealmente supervisado. El personal de la recepción debe estar aislado, con mascarilla, guantes y de ser posible con la implementación de una pantalla plástica de separación. El acompañante del paciente debe esperar idealmente en su vehículo y si no procede, en la sala de espera con el distanciamiento apropiado en caso de haber una o más personas. Una vez en la sala de procedimientos el paciente deberá enjuagarse con una dilución de peróxido de hidrógeno de 10 volúmenes con agua destilada en proporción 1:2 lo que permite contribuir a disminuir la carga viral en la cavidad oral (Sociedad de Ortodoncia y Ortopedia Dentomaxilofacial de Chile).

Por su parte, el odontólogo, deberá vestir con chaqueta o traje de recambio diario de manga larga, con puño cerrado, cuello alto o redondo y por encima 


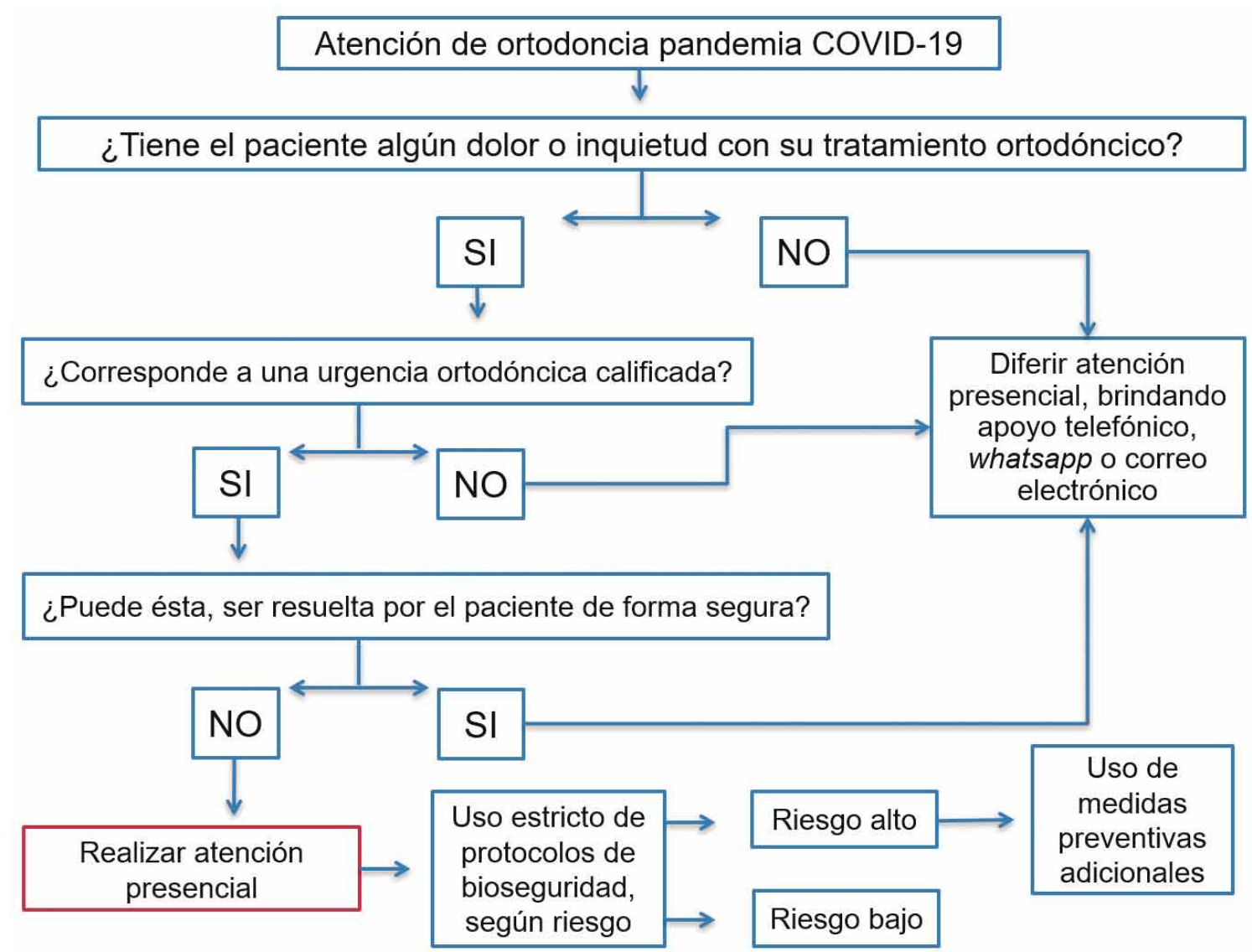

Fig. 1. Algoritmo de atención de pacientes en tratamiento de ortodoncia durante la pandemia COVID-19.

un traje desechable de papel o plástico, el cual deberá ser cambiado con cada paciente. Al igual que en condiciones de no pandemia, es necesario la utilización de mascarilla cubriendo boca y nariz, gorro de recambio diario, guantes desechables y antiparras.

El área clínica deberá contar con el mínimo equipamiento para facilitar la limpieza y desinfección. Se sugiere retirar algodoneros o basureros de la zona de trabajo y cubrir con film descartable la jeringa triple, lámpara y el panel de control del sillón. Para la limpieza de superficies, instrumental y alicates deberán utilizarse desinfectantes de nivel intermedio, que estén registrados en la Environmental Protection Agency (EPA), siguiendo las indicaciones del fabricante y que contengan, dentro de sus químicos, de preferencia alcohol o peróxido de hidrógeno acelerado. Las antiparras deben ser lavadas con agua y jabón antes de reutilizarlas.

El empleo de instrumental rotatorio no está recomendado, por lo tanto debería mantenerse guardado. Dado el caso en que sea inevitable el uso de instrumental generador de aerosol se sugiere aumentar las medidas de protección para disminuir el riesgo de contaminación: utilizar pantalla de cobertura facial completa, mascarilla №95 de uso médico (que permite un mejor sellado), y el trabajo a cuatro manos para que el asistente dental, mediante la cánula de succión, vaya aspirando de forma inmediata.

Alto riesgo. Para la atención de estos pacientes de alto riesgo se incorporan medidas adicionales. En este caso, el paciente deberá ser ingresado de forma inmediata a la sala de procedimientos, avisando previamente su llegada y deberá esperar hasta que le confirmen su ingreso a la consulta. Estos pacientes deberán ser atendidos idealmente en un box único, con puerta cerrada o incluso en su propio auto si lo posee y la urgencia lo permite. Adicional al traje de recambio diario y al desechable por paciente, deberá usarse de forma obligatoria una pechera plástica. Independiente de si se use o no instrumental rotatorio, se debe utilizar pantalla con cobertura facial completa y mascarilla $\mathrm{N}^{\circ} 95$, en caso contrario usar doble mascarilla, ambas de uso único por paciente y el gorro debe ser 
cambiado posterior a la atención del paciente. Los alicates deberán estar estériles y ser de uso único. Se debe evitar al máximo el uso de instrumental rotatorio dada la permanencia probable de hasta tres horas del virus en los aerosoles.

\section{CONCLUSIÓN}

En la medida que los requerimientos lo permitan, se debe preferir siempre la atención remota durante la pandemia COVID-19, siendo importante considerar la ansiedad involucrada en cada paciente debido a la interrupción del tratamiento ortodóncico y las circunstancias presentes. Se debe brindar un buen método remoto de apoyo y seguimiento, lo que podría ser efectivo para el alivio de la inquietud y a futuro posibilitar que el tratamiento concluya de la mejor manera, una vez que la contingencia lo permita.

Si se está frente a un caso real de urgencia ortodóncica calificada que no pueda ser manejada vía remota, se recomienda implementar los protocolos de bioseguridad para la protección de piel y mucosas de manera estricta y rigurosa, teniendo en consideración el riesgo del paciente, recomendándose que firme un consentimiento informado que señala que está en conocimiento de la situación de pandemia y los riesgos que implica.

Para frenar la transmisión del nuevo coronavirus, es esencial aplicar protocolos previos a la atención para clasificar las situaciones que presenten los pacientes en tratamiento de ortodoncia y determinar la conducta a seguir, ante lo cual, resulta de gran utilidad el uso del algoritmo presentado en este artículo. Por otra parte, dado que el profesional especialista es el encargado de evaluar y hacer el manejo de la urgencia, el sentido común debe prevalecer al momento de tomar la decisión, siendo fundamentales aspectos formativos éticos y profesionales.

MATUS, A. C. P.; NEMETH, K. M. E. \& INOSTROZA, T. M. A. Care of patients in orthodontic treatment during the Covid19 pandemic (SARS-CoV-2). Algorithm presentation. Int. J. Odontostomat., 14(4):489-494, 2020.

ABSTRACT. The coronaviruses are an extensive family of viruses that can cause infections both in humans as in animals. The new coronavirus (SARS-CoV2) can cause the disease COVID-19, that was originated in Wuhan , China and it characterizes because of its high rate of contagion among people, which led to the declaration of emergency in public health of international concern in January 2020. The patients who are under orthodontic treatment can present different situations that require attention from this specific area, therefore producing a situation of close contact between patient and dentist with high risk of eminent contagion of SARS-CoV-2. Under this situation, it is necessary that the practice of orthodontics implements prevention protocols, that lead to a correct anamnesis of the patient remotely in order to establish the level of urgency in orthodontics. In case that it cannot be deferred it should be done under strict personal protection measures. The aim of this article is to disclose an algorithm that gives update recommendations for the attention of patients under orthodontic treatment during the COVID-19 pandemic, in order to reduce the possibility of contagion and spread of the disease.

KEY WORDS: COVID-19, Orthodontic treatment, Orthodontics, SARS-CoV-2.

\section{REFERENCIAS BIBLIOGRÁFICAS}

Caprioglio, A.; Pizzetti, G. B.; Zecca, P. A.; Fastuca, R.; Maino, G. \& Nanda, R. Management of orthodontic emergencies during 2019NCOV. Prog. Orthod., 21:10, 2020.

Colegio Médico de Chile \& Sociedad Chilena de Infectología. COVID19. Información Relevante para Personal Sanitario. Santiago de Chile, Colegio Médico de Chile, Sociedad Chilena de Infectología, 2020. Disponible en: http://www.colegiomedico.cl/wp-content/ uploads/2020/03/Informacion_relevante_personal_sanitario.pdf

Gupta, R.; Hussain, A. \& Misra, A. Diabetes and COVID-19: evidence, current status and unanswered research questions. Eur. J. Clin. Nutr., 2020. DOI: https://www.doi.org/10.1038/s41430-020-0652-1

Guo, J.; Xie, H.; Liang, M. \& Wu, H. COVID-19: a novel coronavirus and a novel challenge for oral healthcare. Clin. Oral Invest., 2020. DOI: https://www.doi.org/10.1007/s00784-020-03291-8

Meng, L.; Hua, F. \& Bian, Z. Coronavirus disease 2019 (COVID-19): emerging and future challenges for dental and oral medicine. $J$. Dent. Res., 99(5):481-7, 2020.

Ministerio de Salud (MINSAL). Informe Epidemiológico No1. Enfermedad por SARS-CoV-2 (COVID-19). Santiago de Chile, Ministerio de Salud, Gobierno de Chile, 2020a. Disponible en: https:/ /www.minsal.cl/wp-content/uploads/2020/03/ INFORME_EPI_COVID19_20200330.pdf

Ministerio de Salud (MINSAL). Informe Epidemiológico No16. Enfermedad por SARS-CoV-2 (COVID-19). Santiago de Chile, Ministerio de Salud, Gobierno de Chile, 2020b. Disponible en: https:/ /www.minsal.cl/wp-content/uploads/2020/05/Informe-EPI110520.pdf

Ministerio de Salud (MINSAL). Orientaciones para Atención Odontológica en Fase 4 COVID-19. Santiago de Chile, Ministerio de Salud, Gobierno de Chile, 2020c. Disponible en: https:// diprece.minsal.cl/wp-content/uploads/2020/03/ORIENTACIONES-ATENCION-ODONTOLOGICAS-COVID-19-.pdf

Organización Panamericana de la Salud (OPS). Presentación: Prevención y Control de Infecciones y Nuevo Coronavirus (COVID19): Precauciones Estándares y Uso de Equipos de Protección Personal. Washington (D.C.), Organización Panamericana de la Salud, 2020. Disponible en: https://www.paho.org/es/documen- 
MATUS, A. C. P.; NEMETH, K. M. E. \& INOSTROZA, T. M. A. Atención de pacientes en tratamiento de ortodoncia durante la pandemia COVID-19 (SARS-CoV-2). Presentación de un algoritmo. Int. J. Odontostomat., 14(4):489-494, 2020.

tos/presentacion-prevencion-control-infecciones-nuevocoronavirus-covid-19-precauciones

Sociedad de Ortodoncia y Ortopedia Dentomaxilofacial de Chile (SORTCH). Protocolo de Bioseguridad Sugerido para Atención en Ortodoncia durante Período de Pandemia. Santiago de Chile, Sociedad de Ortodoncia y Ortopedia Dentomaxilofacial de Chile, 2020. Disponible en: http://www.colegiodentistas.cl/inicio/ wp-content/ uploads/2020/04/Protocolo-de-bioseguridad-sugerido-para- atenci\%C3\%B3n-en-ortodoncia-durante-periodo-depandemia.pdf

Volgenant, C. M. C.; Persoon, I. F.; de Ruijter, R. A. G. \& de Soet, J. $\mathrm{J}$. Infection control in dental health care during and after the SARS-CoV-2 outbreak. Oral Dis., 2020. DOI: https://www.doi.org/ 10.1111/odi.13408

World Health Organization (WHO). Coronavirus. Geneva, World Health Organization, 2020a. Disponible en: https://www.who.int/ health-topics/coronavirus\#tab=tab 1

World Health Organization (WHO). Coronavirus Disease 2019 (COVID-19): Situation Report - 120 (19 May 2020). Geneva, World Health Organization, 2020b. Disponible en: https:// www.who.int/docs/default-source/coronaviruse/situation-reports/ 20200519-covid-19-sitrep-120.pdf?sfvrsn=515cabfb_2
Dirección para correspondencia:

Carolina Matus Abásolo

CESFAM Rienzi Valencia

Arturo Prat 1734

Rengo, Sexta Región

CHILE

Email: carolina.matus@ug.uchile.cl

Recibido : 20-05-2020

Aceptado: 22-05-2020 